Supplementary Information

\title{
Analysis of PDA dose curves for the extraction of antimicrobial peptide properties
}

\author{
Jiangtao Zhao, ${ }^{1}$ Kaori Sugihara ${ }^{1,2 *}$
}

${ }^{1}$ Department of Physical Chemistry, University of Geneva, Quai Ernest Ansermet 30, 1211 Geneva 4, Switzerland

${ }^{2}$ Institute of Industrial Science, The University of Tokyo, 4-6-1 Komaba Meguro-Ku, Tokyo 1538505, Japan

*email: kaori-s@iis.u-tokyo.ac.jp

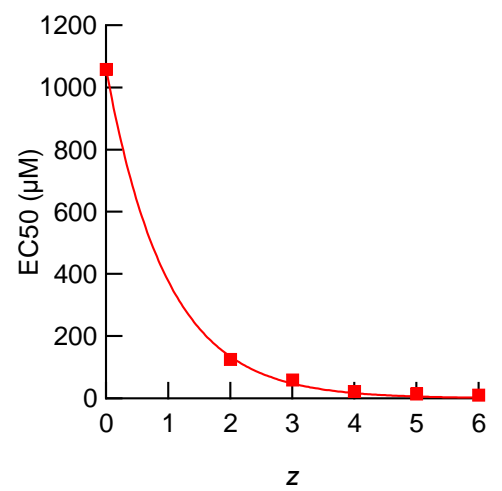

Figure S1: Fitting of Fig. $1 \mathrm{~d}$ in the main manuscript with eq. 1'. Note that at $z=0$ the two values from CHAPS and $\mathrm{C}_{12} \mathrm{E}_{8}$ were averaged $(1058.4 \mathrm{mM})$ and fitted. 

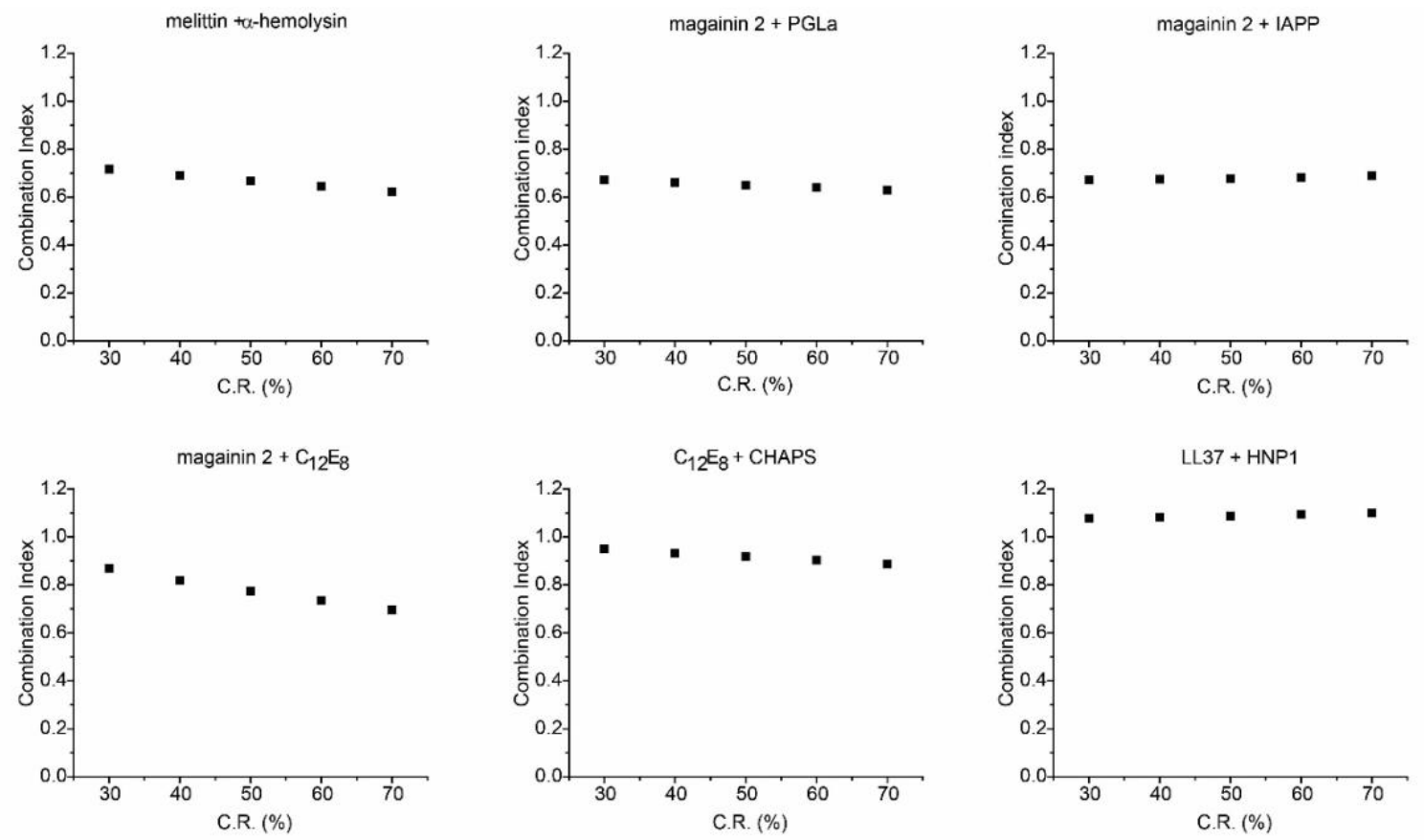

Figure S2: The calculated Combination Index (CI) of various couples of peptides and detergents at different Colorimetrical Response. For a given C.R., the $C I$ was extracted from $C I=\frac{a}{A}+\frac{b}{B}$ (see Materials and Methods in details). The $C I$ of each couple in Table 1 was obtained by averaging these values between $30-70 \%$ together with the standard deviation. 


\section{Materials and methods}

\section{HEPES buffer solution}

All the experiments were conducted in a HEPES buffer solution at $\mathrm{pH} 7.4$, composed of $10 \mathrm{mM}$ 4-(2hydroxyethyl) piperazine-1-ethanesulfonic acid (HEPES, CAS No.:7365-45-9) and $150 \mathrm{mM}$ sodium chloride (CAS No.: 7647-14-5, Roth, Germany) in ultra-pure water filtered through Milli-Q gradient A10 filters (Millipore AG, Switzerland). $\mathrm{pH}$ was adjusted by titrating with $6 \mathrm{mM}$ sodium hydroxide solution (CAS No.:1310-73-2, Sigma-Aldrich Chemie GmbH, Switzerland). The buffer solution was chosen to mimic the peptide-vesicle interactions under physiological conditions, as the ionic strength affects the zeta potential of molecules and thus their binding.

\section{Fabrication of TRCDA/DOPC vesicles}

10,12-Tricosadiynoic acid (TRCDA, CAS No: 66990-30-5) was purchased from Sigma-Aldrich, USA and Alfa Aesar, USA. 1,2-dioleoylsn-glycero-3-phosphocholine (DOPC, CAS No: 4235-95-4) was purchased from Avanti Polar Lipid, USA. To inhibit the spontaneous polymerization, all of the lipids were stored in a freezer $\left(T=-26^{\circ} \mathrm{C}\right)$ as a powder under nitrogen atmosphere (Nitrogen 4.5, PanGas, Lot. 756311-00179757, CH). In prior to experiments, they were dissolved in chloroform (HPLC Grade, Fisher Chemical, USA) at the concentration of $25 \mathrm{mg} / \mathrm{ml}$ and were used within 4 months. To form PDA vesicles, 75\% TRCDA and 25\% DOPC (in a molar percentage) dissolved in chloroform were mixed in a glass vial, dried under nitrogen steam, vacuumed at 400 mbar for 12 hours to entirely remove the chloroform residuals, rehydrated with Milli-Q water at $1 \mathrm{mM}$, followed by vortex for $30 \mathrm{~s}$ and continuous high-power probe sonication (Omni Sonic Ruptor 400 Ultrasonic Homogenizer, Omni International) for $30 \mathrm{~min}$. These samples were kept in a fridge at $4{ }^{\circ} \mathrm{C}$ overnight. This resting time facilitates the following polymerization and allows titanium particles created by the high-power prove sonication to sediment. Supernatant of these samples were was used for the following experiments.

\section{Polymerization of TRCDA/DOPC vesicles}

The supernatant of vesicle suspension was transferred into a plastic tube and was polymerized in a UV box (CL-1000 crosslink, UVP, USA) with the UV dose $0.2 \mathrm{~J} / \mathrm{cm}^{2}$. 


\section{Experiments with peptides}

All the peptides were aliquoted in Milli-Q at different concentrations and stored in freezer $\left(T=-26^{\circ} \mathrm{C}\right)$. Melittin from honey bee venom (CAS No: 0449-79-0) and islet amyloid polypeptide (IAPP, CAS No: 122384-88-7) were purchased from GenScript, USA. Magainin 2 (CAS No: 108433-95-0) was purchased from Biobyt Ltd, UK and Sigma-Aldrich, USA. $\alpha$-hemolysin (CAS No: 94716-94-6), 3-[(3Cholamidopropyl) dimethylammonio]-1 propane sulfonate (CHAPS, CAS No: 75621-03-3) and Dodecyl octaethylene glycol ether $\left(\mathrm{C}_{12} \mathrm{E}_{8}\right.$, CAS Number 3055-98-9) were purchased from SigmaAldrich, USA and Alfa Aesar, USA. LL37 (CAS No: 154947-66-7) was purchased from Peptide Sciences, USA. HNP1(CAS No: 99287-08-8) was purchased from Gls Biochem Shanghai, China. Once the peptides were thawed up, they were stored in a fridge $\left(4{ }^{\circ} \mathrm{C}\right)$ and were used within a couple of days. First, peptide solution at various concentrations was made by sequential dilution with HEPES buffer solution and $66.7 \mu \mathrm{l}$ of each solution was placed in 96 well plate. Next, $10 \mu 1$ of $1 \mathrm{mM}$ blue-state PDA suspension made of MiliQ water and TRCDA/DOPC was added to each well at the final PDA concentration of $130 \mu \mathrm{M}$. The final HEPES and $\mathrm{NaCl}$ concentration is $10 \mathrm{mM}$ and $150 \mathrm{mM}$ respectively for all the wells and the $\mathrm{pH}$ is kept at 7.4. This enables us to confirm that the observed colorimetric response is due to the PDA-peptide interaction but not the slight difference in the salt concentrations or $\mathrm{pH}$. For the mixture of peptides, the molar ratio was always kept at 1:1. These samples were incubated for 5-6 hours at room temperature before measurements.

\section{UV-Vis spectroscopy}

UV-Vis absorption spectra were measured by multi-mode microplate reader (Synergy H1, BioTek, USA) in 96 well-plate. The measurements were carried out with Xenon flash light source. Signals were detected between $300-800 \mathrm{~nm}$ with $1 \mathrm{~nm}$ spacing resolution.

\section{Dynamic light scattering (DLS)}

The particle size was characterized by dynamic light scattering (DLS), using a Nano ZS ZETA SIZER (Malvern Panalytical Ltd) instrument, with classical $90^{\circ}$ dynamic light scattering. All of the experiments were conducted at $25^{\circ} \mathrm{C}$. Vesicle suspensions were diluted 10 folds with HEPES buffer 
solution and introduced into a disposable cuvette made of polystyrene (GLASWARENFABRIK KARL HECHT GMBH \& CO KG, Germany) before the experiments. Each sample was measured three times, and the data was presented as mean \pm standard deviation.

\section{Colorimetric response}

There are two main peaks that are corresponding to blue and red state PDA in UV-Vis absorption spectra. The absorption band of blue and red state PDA are located at around $645 \mathrm{~nm}\left(\lambda_{\text {blue }}\right)$ and $545 \mathrm{~nm}$ ( $\left.\lambda_{\text {blue }}\right)$, respectively. Colorimetric response (C.R.) is a ratiometric analysis between blue and red peaks in PDA that quantifies the transition ${ }^{1,2}$. First, the relative intensity (RI) was described as follows:

$$
R I=\frac{I_{\lambda_{\text {blue }}}}{I_{\lambda_{\text {blue }}}+I_{\lambda_{\text {red }}}}
$$

$I_{\lambda_{\text {blue }}}$ and $I_{\lambda_{\text {red }}}$ are the absorption intensity at the wavelength $\lambda_{\text {blue }}$ and $\lambda_{\text {red. }}$ Next, we used $R I$ to calculate the colorimetric response.

$$
\text { C.R. }(\%)=\frac{R I_{\lambda_{\text {blue }}}-R I_{0}}{R I_{\lambda_{\text {blue }}}} \times 100
$$

$R I_{0}$ is the relative intensity of the control group without addition of peptides. $C . R .=0$ means the absence of the transition and C.R. $=100$ demonstrates that all of the blue state PDA transitioned into red. The data was processed with OriginPro 9.1. After the subtraction of the baseline and calculation of colorimetric response, the dose curves (C.R. vs concentration) were plotted and $E C_{50}$ and Hill coefficients were extracted by fitting with a sigmoidal function:

$$
y=V_{\max } \frac{x^{\alpha}}{E C_{50}^{\alpha}+x^{\alpha}}
$$

$V_{\max }(=100 \%)$ is the maximum colorimetric response, $E C_{50}$ is a half maximum concentration, $\alpha$ is the Hill coefficient, and $y, x$ are the $C . R$. and the ligand concentrations, respectively.

\section{Combination Index (CI)}

Combination index $(\mathrm{CI})$ is a parameter that quantifies the cooperative effect between two types of peptides and can be calculated at an arbitrary response value (in our case C.R.) ${ }^{3,4}$ : 


$$
C I=\frac{a}{A}+\frac{b}{B}
$$

For example, if we measured 3 PDA dose curves against peptide 1, peptide 2, and their 1:1 mixture, and want to calculate $C I$ at $C . R .=50 \%, A$ is the concentration of peptide 1 that induced $C \cdot R=50 \% . B$ corresponds to the concentration of peptide 2 giving $C . R .=50 \%$. Whereas $a, b$ is the concentration of peptide 1 and 2 in the peptide mixture that yielded C.R. $=50 \%$ in the dose curve of the peptide mixture.

\section{References}

1. Rozner, S.; Kolusheva, S.; Cohen, Z.; Dowhan, W.; Eichler, J.; Jelinek, R. Analytical Biochemistry 2003, 319, (1), 96-104.

2. Charych, D.; Nagy, J.; Spevak, W.; Bednarski, M. Science 1993, 261, (5121), 585-588.

3. Foucquier, J.; Guedj, M. Pharmacol Res Perspect 2015, 3, (3), e00149.

4. Patel, H.; Huynh, Q.; Barlehner, D.; Heerklotz, H. Biophys J 2014, 106, (10), 2115-25. 